\title{
GENERATION OF SOUND FROM PRIMORDIAL COSMIC TURBULENCE
}

\author{
HIDEKAZU NARIAI \\ Research Institute for Theoretical Physics, Hiroshima University, Takehara, Japan
}

\begin{abstract}
From the standpoint of the turbulent origin of the formation of galaxies, it is important whether the primordial cosmic turbulence can survive up to the necessary epoch against acoustic decay. This is a preliminary report of our new formalism for attacking the problem.
\end{abstract}

The idea that protogalaxies are formed from primordial turbulence in an expanding universe was originally proposed by von Weizsäcker (1948) and Gamow (1952), and several specific features of cosmic turbulence were studied by the author (1956a, 1956b). After the discovery of the $3 \mathrm{~K}$ background radiation, this idea has been revived by Ozernoy's group $(1968,1969,1971)$ and our group in Japan (Sato et al., 1970, 1971, 1972; Tomita et al., 1970; Nariai and Tomita, 1971; Nariai, 1970, 1971; Tomita, 1971, 1972a, b; Nariai and Fujimoto, 1972), while Zel'dovich (1970), Peebles (1971), and Sunyaev and Zel'dovich (1972) have favoured the density perturbation hypothesis.

On the other hand, Jones (1973) has recently criticized the revived idea on the ground that cosmic turbulence cannot be supported against decay because of the generation of sound after the epoch $t_{*}$ at which the matter density is equal to the radiation density. His assertion is based on the Lighthill (1952) - Crighton (1969) theory for the generation of sound from a turbulent static medium, but Matsuda et al. (1973) have shown that this conclusion is erroneous because of his mis-application of the theory to the turbulent expanding medium. It seems, however, that the cosmic expansion results in more fundamental inadequacies in the applicability of the theory to the present problem, just as it affects the applicability of Jeans's exponential growth-rate for the density contrast due to gravitational instability in an expanding universe.

In the above situation, we have developed a new formalism (Nariai, 1973) for dealing with the generation of sound from primordial cosmic turbulence by constructing the retarded Green's function $D_{\text {ret }}\left(t, \mathbf{x} ; t^{\prime}, \mathbf{x}^{\prime}\right)$ which automatically includes all significant influences of the cosmic expansion upon the propagation of sound waves which are generated. The Green's function shows that acoustic phenomena at a space-time point $(t, \mathbf{x})$ may come not only from points on the acoustic past 'light-cone' $v_{s}\left(t^{\prime}\right)=$ $=v_{s}(t) \exp \left\{2\left|\mathbf{x}-\mathbf{x}^{\prime}\right| / r_{0}\right\}$, but also from points in the acoustic past 'time-like' region $v_{s}\left(t^{\prime}\right)>v_{s}(t) \exp \left\{2\left|\mathbf{x}-\mathbf{x}^{\prime}\right| / r_{0}\right\}$, where $v_{s}(t)=c / \sqrt{3}\{1+3 z(t) / 4\}^{-1 / 2}$ with $z(t) \equiv a(t) / a\left(t_{*}\right)$ $\left(\propto t^{2 / 3}\right.$ or $t^{1 / 2}$ according as the matter density $\varrho_{B m}=\varrho_{B m}^{*} z^{-3}$ is considerably higher or lower than the radiation density $\left.\varrho_{B r}=\varrho_{B r}^{*} z^{-4}\right)$ is the sound velocity and $a\left(t_{*}\right) r_{0} \equiv$ $(4 c / \beta)\left(6 \pi G Q_{B r}^{*}\right)^{-1 / 2}$ with $\beta=1$ (if $\left.z \gg 1\right)$ or $\sqrt{ } \frac{3}{2}$ (if $\left.z \ll 1\right)$. Then the density contrast $K_{m} \equiv \varrho_{m}(t, \mathbf{x}) / \varrho_{B m}(t)-1$ to be acoustically generated from the turbulent velocity field 
$v\left(t^{\prime}, \mathbf{x}^{\prime}\right)$ is given by

$$
\begin{aligned}
K_{m}(t, \mathbf{x})= & \frac{1}{4 \pi v_{s}^{4}(t)} \cdot \frac{a^{2}(t) x_{i} x_{j}}{|\mathbf{x}|^{3}} \int_{r \leqslant r_{m}} \mathrm{~d} \mathbf{x}^{\prime} J\left(r / r_{0}, t\right)\left[\left\{a\left(t^{\prime}\right) /(t)\right\}^{2} \times\right. \\
& \left.\times \frac{\partial^{2}}{\partial t^{\prime 2}}\left\{v_{i}\left(t^{\prime}, \mathbf{x}^{\prime}\right) v_{j}\left(t^{\prime}, \mathbf{x}^{\prime}\right)\right\}\right]_{t^{\prime}=t-h(r, t)},
\end{aligned}
$$

and

$$
J\left(r / r_{0}, t\right) \equiv e^{-9 r / r_{0}}\left[1+\frac{1}{2}\left(r / r_{0}\right)\left\{Z(t) e^{-r / r_{0}}-1\right\}\right]
$$

where $r \equiv\left|\mathbf{x}-\mathbf{x}^{\prime}\right|, r_{m} \sim r_{0} \ln Z(t), Z \equiv(1+3 z / 4)^{1 / 4}$ and $h(r, t)$ is the retardation function which is reduced to $a(t) \sigma(r) / v_{s}(t)$ with $\sigma(r) \equiv\left(r_{0} / 6\right)\left\{1-\exp \left(-6 r / r_{0}\right)\right\}$ if $z \gtrsim 10$. The appearance of the strong convergence factor $J\left(r / r_{0}, t\right)\left(\rightarrow 1\right.$ for $\left.r_{0} \rightarrow \infty\right)$ and the inequality $r \leqslant r_{m}$ is of course due to the above mentioned property of our retarded Green's function.

By a procedure similar to that in the Lighthill-Crighton theory, we can derive from Equation (1) expressions for the intensity of sound and the acoustic power output per unit mass, etc. In particular, it is shown that the maximal physical radius $l_{t} \equiv a(t) \sigma\left(r_{m}\right)$ of a spherical turbulent region is equal to the acoustic event horizon $l_{s} \equiv v_{s}(t) t$. This means that the retardation effect is essential in the evaluation of the intensity of sound at the center of a turbulent fluid sphere, an effect which was discarded in the Crighton theory. Since the above mentioned effects of cosmic expansion on $K_{m}(t, x)$ and related quantities are all significant, the problem whether primordial cosmic turbulence can survive against acoustic decay must be studied in more detail on the basis of our formalism.

It is to be noticed that the Green's function in Silk and Ames's approach (1973) to the density contrast on the scale of galaxies that would arise through cosmic incompressible turbulence is entirely different from ours, because it is merely a formal device to solve an ordinary differential equation for the time-dependent factor of the density contrast without taking account of the retardation effect mentioned above. In other words, their approach is similar to Sasao's (1973).

Note added in proof. The underlying condition of Equation (1) (common to that of Lighthill's corresponding equation in the case of a turbulent static medium) that the observation point is far from the source region is opposite to the real situation in the cosmological problem. In view of this, we (Nariai, 1974) have recently revised the above formalism to derive the required intensity formula free from such a defect.

\section{References}

Crighton, D. G.: 1969, Proc. Camb. Phil. Soc. 65, 557.

Gamow, G.: 1952, Phys. Rev. 86, 251.

Jones, B. J. J.: 1973, Astrophys. J. 181, 269.

Lighthill, M. J.: 1952, Proc. Roy. Soc. A211, 564. 
Matsuda, T., Takeda, H., and Sato, H.: 1973, Prog. Theor. Phys. 49, 1770.

Nariai, H.: 1956a, Sci. Rep. Tohoku Univ. 39, 213.

Nariai, H.: 1956b, Sci. Rep. Tohoku Univ. 40, 40.

Nariai, H.: 1970, Prog. Theor. Phys. 44, 110.

Nariai, H.: 1971, Prog. Theor. Phys. 45, 61.

Nariai, H.: 1973, Prog. Theor. Phys. 50, in press.

Nariai, H. : 1974, Prog. Theor. Phys. 51, 5, in press.

Nariai, H. and Tomita, K.: 1971, Prog. Theor. Phys. Suppl., No. 49, p. 83.

Nariai, H. and Fujimoto, M.: 1972, Prog. Theor. Phys. $47,105$.

Ozernoy, L. M. and Chernin, A. D.: 1968, Soviet Astron. AJ 11, 907.

Ozernoy, L. M. and Chernin, A. D.: 1969, Soviet Astron. AJ 12, 901.

Ozernoy, L. M. and Chibisov, G. V.: 1971, Soviet Astron. AJ 14, 615.

Peebles, P. J. E.: 1971, Astrophys. Space Sci. 11, 443.

Sasao, T.: 1973, Publ. Astron. Soc. Japan 25, 1.

Sato, H., Matsuda, T., and Takeda, H.: 1970, Prog. Theor. Phys. 43, 1115.

Sato, H., Matsuda, T., and Takeda, H.: 1971, Prog. Theor. Phys. Suppl., No. 49, p. 11.

Sato, H., Matsuda, T., and Takeda, H.: 1972, Prog. Theor. Phys. 48, 1503.

Silk, J. and Ames, S. : 1973, Astrophys. J. 178, 77.

Sunyaev, R. A. and Zel'dovich, Ya. B.: 1972, Astron. Astrophys. 20, 189.

Tomita, K.: 1971, Prog. Theor. Phys. 45, 1747.

Tomita, K.: 1972a, Prog. Theor. Phys. 47, 416.

Tomita, K.: 1972b, Prog. Theor. Phys. 48, 1503.

Tomita, K., Nariai, H., Sato, H., Matsuda, T., and Takeda, H.: 1970, Prog. Theor. Phys. 43, 1511.

Von Weizsäcker, C. F.: 1948, Z. Astrophys. 24, 181.

Zel'dovich, Ya. B. : 1970, Astron. Astrophys. 5, 84.

\section{DISCUSSION}

Ozernoy: The inhomogeneities associated with sound, are of two kinds: those of local origin (for them $\delta \equiv\left(\overline{\delta \varrho^{2}} / \varrho^{2}\right)^{1 / 2} \sim M^{2}, M$ being the Mach number) and inhomogeneities produced by the emission of sound. The generation of the latter was investigated by Dr A. Kurskov of our group in great detail. He found that on the maximum scale of cosmological turbulence their amplitude does not exceed $\delta^{2} \sim M^{7}$ at the moment of equal matter and radiation densities. This is $\left(R_{e} / M^{2}\right)_{\mathrm{eq}} \sim 7 \times 10^{6}\left(\Omega h^{2}\right)^{2}$ times less than the value $\delta^{2} \sim M^{5} R_{e}$ (where $R_{e}$ is Reynolds number) obtained by B. Jones. The difference is due to the fact that this amplitude from the Lighthill-Crighton theory used by Jones is valid only for established stationary turbulence and cannot be attained in expanding cosmological turbulence.

The energy content of the sound generated by cosmological turbulence turns out to be negligibly small as compared with the energy of the turbulence itself. Therefore the dissipation of turbulence through the generation of sound as well as the role of these sound waves for the cosmogonical purposes has been significantly overestimated. Dr Susan Ames kindly informed me at this Symposium that taking into account the damping of sound waves also diminishes their efficiency in galaxy formation. 